Relations industrielles

Industrial Relations

\title{
Psychologie de la formation, jalons et perspectives par Jacques \\ LEPLAT, Toulouse : Octarès, 2002, 293 p., ISBN 2-906769-83-5.
}

\section{Pierre-Sébastien Fournier}

Volume 60, numéro 2, printemps 2005

URI : https://id.erudit.org/iderudit/011727ar

DOI : https://doi.org/10.7202/011727ar

Aller au sommaire du numéro

Éditeur(s)

Département des relations industrielles de l'Université Laval

ISSN

0034-379X (imprimé)

1703-8138 (numérique)

Découvrir la revue

Citer ce compte rendu

Fournier, P.-S. (2005). Compte rendu de [Psychologie de la formation, jalons

et perspectives par Jacques LEPLAT, Toulouse : Octarès, 2002, 293 p., ISBN

2-906769-83-5.] Relations industrielles / Industrial Relations, 60(2), 375-376.

https://doi.org/10.7202/011727ar d'utilisation que vous pouvez consulter en ligne.

https://apropos.erudit.org/fr/usagers/politique-dutilisation/ 
a basic human right may have been the driving force for broader dissemination of the original report in book form, but Campa's other analytic and descriptive contributions also justify its re-issue. Unfair Advantage is a valuable addition to the literature for both the specialist and the general reader.

\author{
LAWRENCE S. ROOT \\ University of Michigan
}

\section{Psychologie de la formation, jalons et perspectives}

par Jacques LEPLAT, Toulouse : Octarès, 2002, 293 p., ISBN 2-906769-83-5.

$\mathrm{Au}$ cours de sa carrière, Jacques Leplat a contribué de façon significative au développement de l'ergonomie et de la psychologie du travail française. Cet ouvrage rassemble diverses contributions de l'auteur au développement de la formation depuis les années cinquante. Ces textes sont regroupés autour de six thèmes pertinents : 1) vues d'ensemble sur les aspects psychologiques de la formation professionnelle; 2) l'analyse de l'activité en vue de la formation; 3 ) la dimension temporelle dans le travail et dans la formation: le cas du travail manuel; 4) un principe majeur de la formation : la connaissance des résultats; 5) la formation comme acquisition d'habiletés; et 6) une méthode d'assistance à la formation : l'enseignement programmé.

Pour chacun de ces thèmes, l'auteur regroupe des textes rédigés entre 1955 et 1998. Un tel exercice permet notamment de saisir l'évolution des idées et des perspectives de la formation. Cette réflexion permet également de mieux saisir les bases théoriques et méthodologiques des travaux contemporains sur la formation en psychologie du travail mais également en ergonomie française.

La thématique abordant les vues d'ensemble sur les aspects psychologiques de la formation professionnelle se compose de deux textes (1955 et 1992). Le premier (1955) pose le problème de la formation et de la contribution de la psychologie au contenu ainsi qu'à sa structure. L'auteur voyait alors l'apport possible de la psychologie comme outil efficace pour surmonter les difficultés de la formation. Le second texte (1992) se positionne résolument dans une perspective de la psychologie cognitive pour contribuer à l'élaboration d'une formation. La discussion porte sur quatre phases d'élaboration d'un programme de formation: l'étude des conditions initiales, le diagnostic des problèmes de formation, l'élaboration et la mise en œuvre de la formation et, finalement, l'évaluation des résultats. L'étude des conditions s'illustre par deux questions : «formation de qui ?» et «formation à quoi ? ». Le diagnostic vise à documenter les caractéristiques des formés, les compétences requises pour une tâche et les compétences à acquérir. L'élaboration de la formation concerne la conception du contenu et des méthodes ainsi que la réalisation de la formation. Finalement, l'évaluation des résultats vise à mesurer l'atteinte des objectifs et l'utilité de la formation.

La thématique de l'analyse de l'activité en vue de la formation se compose également de deux textes (1955 et 1998). Le premier (1955) montre la pertinence de « l'analyse du travail » pour la formation. Par le biais d'une démarche expérimentale, l'auteur montre la pertinence des résultats et d'une méthode d'analyse du travail pour contribuer à la formation. Le second texte (1998) se situe dans un contexte d'activités physiques et sportives où l'auteur discute de la pertinence de l'analyse de l'activité en ergonomie pour comprendre une activité et pour la transformer. Cette réflexion met en évidence des préoccupations communes 
avec les sciences de l'activité sportive et ouvre sur des intérêts réciproques.

La thématique dimension temporelle dans le travail et dans la formation : le cas du travail manuel comporte deux textes (1956 et 1970). Le premier (1956) se veut une critique de l'étude des temps et mouvements largement répandue à cette époque. Alors que cette méthode implique une décomposition de l'action, et par le fait même une parcellisation des tâches, l'auteur montre que l'action doit être considérée comme un tout organisé qui ne peut se réduire à une évaluation en temps et mouvements. Le second texte (1970) s'intéresse à l'évaluation de la vitesse d'exécution pour mesurer l'apprentissage. À travers la littérature, Leplat voit la pertinence de ce critère dans l'évaluation de l'apprentissage mais constate qu'elle ne devrait cependant pas être priorisée.

La thématique sur la connaissance des résultats comme principe majeur de la formation se compose de deux textes (1970 et 1962). La notion de « connaissance des résultats » utilisée par l'auteur s'apparente étroitement aux notions de feed-back, de récompense et de renforcement. Le premier texte (1970) s'intéresse aux différentes formes de la connaissance des résultats, aux modalités d'action de celles-ci ainsi qu'à leur application. Le second texte (1962) présente une recherche sur l'impact de la connaissance des résultats et ouvre sur une utilisation pédagogique.

La thématique formation comme acquisition d'habiletés comporte deux textes (1988 et 1981) situés dans une perspective de psychologie cognitive. Le premier texte (1988) tente de définir la notion d'habileté qui, selon l'auteur, est difficile à cerner puisqu'elle varie selon le contexte théorique utilisé et selon les situations qu'elle désigne. Dans cette perspective, l'auteur tente de définir ses différentes facettes. Le second texte (1981) s'intéresse au rapport entre le développement d'habiletés mentales et les connaissances livresques que l'auteur nomme des «techniques». L'article constate l'importance des techniques dans le développement d'habiletés. Cependant, pour atteindre un haut niveau d'habileté, elles doivent être complétées par la pratique.

La thématique sur l'enseignement programmé s'intéresse aux méthodes et aux technologies d'aide à la formation. Ce texte, rédigé à une époque où l'utilisation de l'ordinateur demeurait limitée, permet de mettre en perspective l'utilisation actuelle des technologies de l'information dans l'enseignement.

En guise de conclusion, Jacques Leplat identifie les thèmes prometteurs pour le développement de la formation et de la «psychologie de la formation ». Il souligne trois perspectives utilisées dans la conception de formation : la didactique professionnelle, la formation expérientielle et la formation selon l'apprentissage situé. Il ouvre également sur les perspectives de recherche qui combinent à la fois activité et formation et, plus particulièrement, l'analyse de l'activité comme contribution à la formation et à ses diverses composantes. Il montre également la pertinence de la connaissance des résultats, de la notion de compétences, de la notion d'activités collectives et de la formation à celles-ci ainsi que de la poursuite du développement des aides à la formation.

Ce recueil de textes rédigés à différents moments de l'évolution du rapport entre l'analyse du travail et la formation en France permet de saisir les jalons de l'évolution historique de ce domaine d'étude. La contribution principale de cet ouvrage se situe alors au plan de la mise en perspective de l'état des connaissances actuelles sur l'analyse du travail dans la formation par rapport à son évolution au cours des cinquante dernières années.

Pierre-Sébastien Fournier Université Laval 